\title{
Análisis de mortalidad debida a una infección del torrente sanguíneo por Enterococcus faecium en Taiwán central
}

\author{
Chang-Hua Chen, Li-Chen Lin, Yu-Jun Chang y Chih-Yen Chang
}

\section{Mortality analysis of Enterococcus faecium bloodstream infection in central Taiwan}

Background: Bloodstream infections (BSIs) due to Enterococcus faecium (E. faecium), particularly those due to vancomycin-resistant enterococcus (VRE), are still a therapeutic challenge. Aim: To evaluate mortality from BSI due to E. faecium and VRE in central Taiwan. Materials and Methods: We retrospectively analyzed cases of significant E. faecium BSI in the Changhua Christian Hospital System between January 1, 2010 and December 31, 2013. Results: Of the 76 cases, 28 patients (36.8\%) were admitted to intensive care units (ICUs) at the onset of BSI, $10(13.2 \%)$ cases were associated with polymicrobial bacteremia, and $29(38.2 \%)$ cases were associated with entry via the biliary tract. VRE was observed in 18 (23.7\%) cases. The 30-day mortality rate was $13.1 \%(10 / 76)$. Multivariate logistic regression analysis showed that bacteremia of non-biliary tract origin $(\mathrm{OR}=8.43,95 \%$ confidence interval $(95 \% \mathrm{CI})=1.32-54.00, \mathrm{p}=0.002)$ and $\mathrm{ICU}$ admission $(\mathrm{OR}=4.2,95 \% \mathrm{CI}=1.7-10.0, \mathrm{p}=0.002)$ were significant risk factors for 30-day mortality, whereas appropriate antimicrobial therapy was a protective factor for 30-day mortality $(\mathrm{OR}=0.33,95 \% \mathrm{CI}=0.14-0.79, \mathrm{p}=0.013)$. Conclusions: Our results underscore the need to assist patients admitted to ICUs with E. faecium BSIs with a non-biliary tract origin. We emphasize the use of appropriate antimicrobial therapy for E. faecium BSI with the aim to rescue more patients with these infections.

Key words: Mortality, vancomycin-resistant enterococcus, bloodstream infection, Enterococcus.

Palabras clave: Enterococcus faecium, enterococos resistente a vancomicina, infección del torrente sanguíneo, mortalidad.

\section{Introducción}

$\mathrm{E}$ 1 manejo de las infecciones del torrente sanguíneo (ITS) debidas a Enterococcus faecium, en particular aquellas por enterococos resistentes a vancomicina (ERV), se ha convertido en un verdadero desafío para lograr un tratamiento adecuado. Las ITS por ERV fueron comunicadas por primera vez en 1988, y poco tiempo después, se reportaron algunos casos en Estados Unidos de América (E.U.A.) y en algunos países europeos. En 1996 se informó del primer paciente con ITS por ERV en Taiwán. En E.U.A., los ERV se han convertido en importantes patógenos nosocomiales y su incidencia ha aumentado de $0,3 \%$ en el año 1988, a $30 \%$ en el 2003 , de acuerdo con la información del Sistema de Vigilancia Nacional de Infecciones Nosocomiales; además, los ERV representaron 14\% de las cepas aisladas de Enterococcus en pacientes ingresados en las Unidades de Cuidados Intensivos (UCI). Aunque los ERV representan menos de $2 \%$ de los enterococos identificados en el Programa de Vigilancia de Resistencia Antimicrobiana en Taiwán en el año 2000, se ha reportado un rápido crecimiento en la resistencia a vancomicina, desde $1,2 \%$ en el año 1996 , a $6,1 \%$ en el 2003 , entre los aislados de enterococos nosocomiales en hospitales de distintas universidades en Taiwán.

La existencia de E. faecium es preocupante debido a las limitadas opciones de tratamiento existentes. De igual manera, los ERV representan un tema clínico importante.

La mayoría de las publicaciones sobre ITS debidas a Enterococcus spp están relacionada con la infección en general, con la geografía de la infección y con la resistencia a vancomicina. Además, la mayoría de los estudios publicados a la fecha se han llevado a cabo en E.U.A., donde las condiciones epidemiológicas pueden ser diferentes a las existentes en Asia. Se han analizado los factores de riesgo asociados a la mortalidad intrahospitalaria por ITS E. faecium, pero la mayoría de éstos son indicadores de ciencias médicas básicas y bio-marcadores moleculares, que no son útiles para los médicos tratantes. Por otra parte, la información correspondiente a ITS $E$. faecium es escasa en Taiwán central.

El objetivo del presente estudio es analizar la incidencia de la ITS causadas por E. faecium y los factores de riesgo de mortalidad por ITS E. faecium en Taiwán central. También nos enfocamos en evaluar la presentación clínica y los resultados de una ITS por ERV.
Hospital Changhua Christian, Taiwan.

Comité de Control de Infecciones (CHC, LCL, CYC).

Departamento de Medicina Interna División de Enfermedades Infecciosas (CHC, CYC) Centro de Epidemiología y Estadística (YC).

Universidad Hung Kuang,

Taiwán.

Escuela de Medicina y Enfermería, Departamento de Enfermería (CHC)

Los autores declaran no tener conflictos de interés

Financiamiento: Hospital Changhua Christian.

El estudio fue aprobado por el Consejo de Revisión Institucional del Hospital Changhua Christian (CCH IRB N 140304).

Recibido: 28 de agosto de 2015 Aceptado: 26 de julio de 2016

Correspondencia a: Chen Changhua chenchanghuachad@gmail.com 


\section{Materiales y Métodos}

\section{Escenario clínico y población en estudio}

La población residente en las áreas rurales del Taiwán central es mayoritariamente atendida en el Sistema del Hospital Changhua Christian (SHCC), que cuenta con 4.000 camas. El Hospital Changhua Christian (HCC) es una de las nueve sucursales de hospitales del SHCC y es un centro médico de derivación de atención terciaria, con 1.800 camas, ubicado en Taiwán central. Este estudio fue llevado a cabo en el SHCC y fue aprobado por el Consejo de Revisión Institucional de HCC. Los casos de ITS E. faecium fueron analizados revisando los registros médicos; se llevó a cabo un estudio retrospectivo de corte transversal entre el 1 de enero de 2010 y el 31 de diciembre de 2013, en el SHCC.

\section{Análisis de los casos}

Los casos de ITS E. faecium fueron identificados a partir de la base de datos microbiológicos y los registros médicos, en concordancia con la Clasificación Internacional de Enfermedades, $9^{\text {a }}$ edición, con algunas modificaciones clínicas (ICD-9-CM), realizadas en el SHCC, en Taiwán central. Utilizamos índices computarizados para detectar casos con los siguientes códigos ICD-9-CM: V09.8, 041.04, 790.7, 038, y 038.9. Todos los pacientes diagnosticados con ITS E. faecium durante el período de estudio fueron incluidos.

\section{Criterios de exclusión}

Edad $<18$ años, información clínica insuficiente, inconsistencia entre la información de los códigos ICD-9 y el conjunto de datos microbiológicos y malinterpretación de los exámenes microbiológicos, incluyendo identificación incorrecta, contaminación bacteriana e información con importancia clínica desconocida.

Para cada caso se efectuó un registro médico, conteniendo diagnósticos, datos de intervenciones quirúrgicas y otra información importante. Los registros médicos de todos los casos con ITS E. faecium fueron revisados manualmente por el investigador principal (C.H.H.) para confirmar el diagnóstico (usando los recursos del SHCC). Los casos clínicamente erróneos fueron registrados por el investigador secundario (C.C.Y). Se incluyó en el análisis estadístico sólo el primer episodio de ITS por $E$. faecium en cada paciente durante el período de estudio. Para calcular la incidencia de enfermedad, fue considerada bajo riesgo de infección la población total de pacientes del SHCC.

\section{Identificación bacteriana y análisis}

Todas las cepas aisladas de E. faecium entre el 1 de enero de 2010 y el 31 de diciembre de 2013 fueron consideradas clínicamente significativas, de la cuales, 18 cepas fueron identificadas como ERV en Taiwán central. Sólo se analizó la primera cepa de cada paciente. La identificación de laboratorio para ERV fue realizada utilizando $\mathrm{NaCl}$ 6,5\% /test de agar bilis-esculina y se confirmó mediante Vitek-2 para especies grampositivas (bioMérieux, Marcy l'Etoile, Francia). Se evaluó la susceptibilidad antimicrobiana in vitro para vancomicina, ampicilina, altas concentraciones de estreptomicina y de gentamicina, teicoplanina y linezolid. Las concentraciones inhibitorias mínimas (CIM) de vancomicina y teicoplanina fueron calculadas utilizando en método de prueba $E$ (AB Biodisk, Solna, Suecia) en agar Mueller-Hinton. Se utilizó los puntos de corte recomendados por Clinical Laboratory Standards Institute para determinar la susceptibilidad de las cepas. La resistencia a vancomicina fue definida como $\mathrm{CIM} \geq 32 \mu \mathrm{g} / \mathrm{mL}$.

\section{Definiciones}

ITS significativa: más de dos hemocultivos positivos con E. faecium o uno solo positivo con E. faecium, asociados a una fuente de infección clínicamente significativa.

Fecha de inicio de una ITS: fecha en la cual el cultivo de la primera muestra de sangre fue positivo.

Bacteriemia polimicrobiana: presencia de más de un microorganismo en un mismo hemocultivo.

La gravedad subyacente fue clasificada de acuerdo a los criterios de McCabe y Jackson. Durante las primeras $24 \mathrm{~h}$ desde la aparición de una ITS por E. faecium, un síndrome de respuesta inflamatoria sistémica (SRIS), sepsis, sepsis grave y shock séptico, definido como fue descrito previamente. En el SHCC se evaluaron los resultados 30 días después de la aparición de una ITS por E. faecium.

Se consideró como muerte asociada a una ITS si el paciente falleció en menos de dos semanas después de la aparición de la ITS, siempre y cuando no se hubiera identificada alguna otra causa de muerte.

El grupo de fallecidos comprende a los pacientes con ITS por E. faecium que fallecieron dentro de los 30 días después de ser diagnosticados.

Se clasificó una ITS por E. faecium de acuerdo a una versión modificada de la descripción de Horan, ya fuese al comienzo de la atención médica o al momento de ser adquirida por el paciente en su comunidad.

El grupo ERV corresponde a los pacientes cuyas cepas de E. faecium mostraron CIM $\geq 32 \mu \mathrm{g} / \mathrm{mL}$ para vancomicina.

Se definió como: tratamiento antimicrobiano apropiado a los pacientes que recibieron antimicrobianos de forma efectiva, así como el ser evaluado con pruebas de susceptibilidad durante el período de la terapia empírica.

\section{Análisis estadístico}

Fue realizado usando la prueba $\chi^{2}$ o la prueba de dos colas exacta de Fisher para variables binarias, y el test 
de Student o el test de Mann-Whitney para la escala de variables continuas.

La información continua fue expresada como el primedio \pm deviación estándar, o como la mediana y el rango. El análisis univariado y multivariado se realizó para determinar los factores de riesgo independientes para una mortalidad a 30 días, todo esto utilizando los modelos de regresión logística. Se consideró como estadísticamente significativo al valor $<0,05$. Todas las pruebas fueron realizadas utilizando el software SPSS, versión 17.0 (SPSS).

\section{Resultados}

\section{Población de estudio}

Durante el período de estudio, de las 1.490 muestras de sangre positivas, $133(7,6 \%)$ arrojaron la presencia de $E$. faecium, ya fuese como único aislado o en combinación con otros microorganismos. Posteriormente, se definió en 92 pacientes que eran una ITS clínicamente significativa, habiendo excluido duplicados de 21 pacientes. Diez y seis pacientes fueron excluidos $\mathrm{y}$, consecuentemente, fueron analizados 76 pacientes (Figura 1). La incidencia de ITS debida a $E$. faecium fue de 0,29 casos por cada 1.000 pacientes ingresados.

\section{Información microbiológica}

A partir de los aislados de E. faecium recolectados de 76 pacientes, 18 fueron ERV (23,7\%). Diez pacientes presentaron una bacteriemia polimicrobiana $(13,2 \%)$. Entre los microorganismos encontrados, se incluyen: Escherichia coli (n: 4), Klebsiella pneumoniae (n: 2), Pseudomonas aeruginosa, Enterobacter aerogenes, Citrobacter freundii y Acinetobacter baumannii (1 paciente por cada uno). Correspondiente a los 10 casos con bacteriemia polimicrobiana, 7 (70\%) no presentaron ERV y 3 sí fueron ERV (30\%). Durante el período de estudio, 18 pacientes fueron identificados con ITS por ERV (23,7\%). La susceptibilidad antimicrobiana de los 18 ERV aislados se muestra en la Tabla 1.

\section{Información epidemiológica y enfermedades subyacentes en los grupos con ERV y sin ERV}

Las características epidemiológicas y las condiciones subyacentes de los 76 pacientes se muestras en la Tabla 2. La distribución de todas las características analizadas no fue significativamente diferente entre el grupo con $\operatorname{ERV}(8 / 76 ; 23,7 \%)$ y el grupo sin ERV (58/76; 76,3\%). Treinta y nueve pacientes fueron hombres $(51,8 \%)$, y la edad promedio fue de 59,1 años (DS 17,8; rango: 21-89 años). La enfermedad subyacente más común fue la enfermedad biliar (75\%), seguida por cáncer de órganos sólidos (44,6\%). De acuerdo con el criterio de McCabe y Jackson, las enfermedades subyacentes no fueron fatales

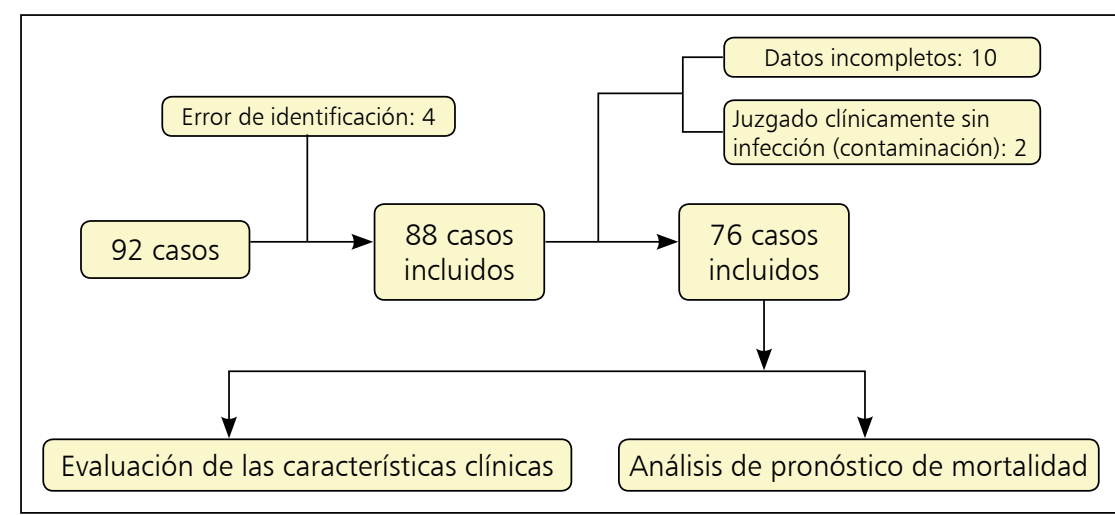

Figura 1.

Tabla 1. Susceptibilidad antimicrobiana de 18 aislados de ERV

\begin{tabular}{lccc}
\hline Antimicrobiano & $\mathrm{CIM}_{\mathbf{5 0}}(\boldsymbol{\mu g} / \mathbf{m L})$ & $\mathrm{CIM}_{\mathbf{9 0}}(\boldsymbol{\mu g} / \mathbf{m L})$ & \\
Ampicilina & 1,5 & 2 & $1-6$ \\
Vancomicina & $>256$ & $>256$ & $>256$ \\
Teicoplanina & 16 & 64 & $8-128$ \\
Gentamicina & 24 & $>1.024$ & $12->1.024$ \\
Streptomicina & $>1.024$ & $>1.024$ & $96->1.024$ \\
Linezolid & 2 & 2 & $1,5-2$ \\
\hline
\end{tabular}

en 41 pacientes $(53,6 \%)$; no obstante, fueron fatales para 34 pacientes $(44,6 \%)$, y rápidamente mortales en un paciente $(1,8 \%)$. Entre los factores que predispusieron a los pacientes a una ITS, 29 pacientes $(37,5 \%)$ tuvieron catéteres de drenaje biliar, $23(30,4 \%)$ tuvieron un historial de ingreso en el hospital, 10 (12,5\%) tuvieron leucopenia y $7(8,9 \%)$ tenían catéteres venosos centrales. Treinta y nueve episodios de ITS ocurrieron en el hospital, con un periodo de internación previo a la aparición de la ITS de 9,0 días (DS 6,8; rango: 3-89 años). Veintiocho pacientes $(36,8 \%)$ estaban en la UCI al momento del comienzo de la ITS.

\section{Análisis de mortalidad a 30 días por ITS causada por E. faecium}

Los resultados están resumidos en la Tabla 3. El rango de mortalidad a 30 días fue de 13,1\% (10 de 76 pacientes). El análisis de regresión logística multivariada (Tabla 4), incluyendo el análisis univariado de variables con $\mathrm{p}<0,05$, mostró que la ITS con un origen no biliar $(\mathrm{OR}=8,43 ; \mathrm{IC} 95 \%=1,32-54,0 ; \mathrm{p}=0,002) \mathrm{y}$ el ingreso a la UCI (OR $=4,2$; IC IC95\% $=1,7-10 ; p=0,002)$ fueron los únicos factores de riesgo para mortalidad a 30 días. El tratamiento adecuado de antimicrobianos fue un factor protector en mortalidad a 30 días $(\mathrm{OR}=0,33$; IC95\% $=0,14-0,79 ; p=0,013)$. 
Tabla 2. Características clínicas y microbiológicas de pacientes con ITS por ERV y no ERV

\begin{tabular}{|c|c|c|c|c|}
\hline Características & No ERV, n: 58 (\%) & ERV, n: $18(\%)$ & Todos, n: 76 (\%) & $\mathbf{p}$ \\
\hline Sexo masculino & $31(53,4)$ & $8(44,4)$ & $39(51,3)$ & 0,672 \\
\hline Edad (años) ( \pm SD) [rango] & $62,1 \pm 9,9[21-89]$ & $58,1 \pm 19,8[40-77]$ & 21-89 & 0,149 \\
\hline \multicolumn{5}{|l|}{ Enfermedades subyacentes o factores de riesgo ${ }^{1}$} \\
\hline Enfermedad biliar & $44(75,9)$ & $13(72,2)$ & $57(75)$ & 0,792 \\
\hline Cáncer de tumor sólido & $28(48,2)$ & $6(33,3)$ & $34(44,7)$ & 0,689 \\
\hline Hipertensión arterial & $8(13,8)$ & $4(22,2)$ & $12(15,8)$ & 0,412 \\
\hline Diabetes mellitus & $7(12,1)$ & $4(22,2)$ & $11(14,5)$ & 0,517 \\
\hline Cirrosis hepática & $3(5,2)$ & $1(5,6)$ & $4(5,3)$ & 0,289 \\
\hline Enfermedad neurológica & 0 & $2(11,1)$ & $2(2,6)$ & - \\
\hline Trauma múltiple & $1(1,7)$ & 0 & $1(1,3)$ & - \\
\hline Neoplasia hematológica & $1(1,7)$ & 0 & $1(1,3)$ & - \\
\hline \multicolumn{5}{|l|}{ Criterio de McCabe y Jackson } \\
\hline Enfermedad no fatal & $27(46,6)$ & $14(77,8)$ & $41(53,9)$ & 0,165 \\
\hline Enfermedad finalmente fatal & $30(51,7)$ & $5(27,8)$ & $34(44,7)$ & 0,412 \\
\hline Enfermedad rápidamente fatal & $1(1,7)$ & 0 & $1(1,3)$ & - \\
\hline \multicolumn{5}{|l|}{ Factores de riesgo' } \\
\hline Catéter de drenaje biliar & $25(43,1)$ & $4(22,2)$ & $29(38,2)$ & 0,415 \\
\hline Previo al ingreso al hospital, dentro de los 6 meses & $17(29,3)$ & $6(33,3)$ & $23(30,3)$ & 1 \\
\hline Leucopenia² $^{2}$ & $8(13,8)$ & $1(5,6)$ & $10(13,2)$ & 0,83 \\
\hline Catéter venoso central & $3(5,2)$ & $4(22,2)$ & $7(9,2)$ & 0,075 \\
\hline Catéter vesical permanente & $1(1,7)$ & $4(22,2)$ & $5(6,6)$ & 0,066 \\
\hline Previo a la Unidad de Cuidados Intensivos ${ }^{3}$ & $1(1,7)$ & $4(22,2)$ & $5(6,6)$ & 0,066 \\
\hline Cirugía reciente $^{3}$ & $1(1,7)$ & $2(11,1)$ & $3(3,9)$ & 0,897 \\
\hline Ventilación mecánica & $1(1,7)$ & $2(11,1)$ & $3(3,9)$ & 0,79 \\
\hline Tratamiento inmunosupresor ${ }^{4}$ & $4(6,9)$ & 0 & $4(5,3)$ & - \\
\hline Sangrado en las dos semanas anteriores & $1(1,7)$ & 0 & $1(1,3)$ & - \\
\hline Quimioterapia por cáncer ${ }^{3}$ & $1(1,7)$ & 0 & $1(1,3)$ & - \\
\hline \multicolumn{5}{|l|}{ Lugar de adquisición de la bacteriemia } \\
\hline Hospital & $31(53,4)$ & $8(44,4)$ & $39(51,3)$ & 0,064 \\
\hline Comunidad & $27(46,5)$ & $10(55,6)$ & $37(48,7)$ & 0,076 \\
\hline \multicolumn{5}{|l|}{ Pabellón al momento de la infección por bacteriemia } \\
\hline Pabellón médico & $30(51,7)$ & $8(44,4)$ & $38(50)$ & 0,059 \\
\hline Sala de emergencias & $20(34,5)$ & $5(27,8)$ & $24(31,6)$ & 0,067 \\
\hline Pabellón de cirugías & $7(12,1)$ & $2(11,1)$ & $9(11,9)$ & 0,78 \\
\hline Unidad médica de cuidados intensivos & $20(34,5)$ & $7(38,9)$ & $27 \quad(3,35)$ & - \\
\hline Unidad de cuidados intensivos de cirugía & $1(1,7)$ & 0 & $1(1,3)$ & - \\
\hline \multicolumn{5}{|l|}{ Tiempo de estadía en el hospital antes de la bacteriemia } \\
\hline Media de días (rango) ${ }^{5}$ & $10,0(3-46)$ & $8,0(3-89)$ & $9,0(3-89)$ & 0,875 \\
\hline Tratamiento antimicrobiano adecuado & $53(91,4)$ & $4(22,2)$ & $67(88,2)$ & 0,025 \\
\hline \multicolumn{5}{|l|}{ Manifestación inicial dentro de las primeras $24 \mathrm{~h}$} \\
\hline Bacteriemia sin SRIS ${ }^{6}$ & $10(17,2)$ & $5(27,8)$ & $15(19,7)$ & 0,418 \\
\hline Sepsis & $40(68,9)$ & $11(61,1)$ & $50(65,8)$ & 0,68 \\
\hline Sepsis grave & $6(10,3)$ & $1(5,5)$ & $7(9,2)$ & 0,79 \\
\hline Shock séptico & $3(5,2)$ & $1(5,5)$ & $4(5,3)$ & 0,789 \\
\hline Bacteriemia polimicrobiana & $8(13,8)$ & $2(11,1)$ & $10(13,2)$ & 0,429 \\
\hline
\end{tabular}


Tabla 3. Pronóstico de los pacientes con ITS debida a E. faecium

\begin{tabular}{|c|c|c|c|}
\hline Características & Grupo sobreviviente, $n=66(\%)$ & Grupo de fallecidos ${ }^{1}, n=10(\%)$ & Valor $p$ \\
\hline Edad (años) ( \pm SD) [rango] & $65,1 \pm 9,9[21-89]$ & $52,1 \pm 19,8[40-77]$ & $0,049 *$ \\
\hline Edad $>60$ años & $29(43,9)$ & $5(50)$ & 0,63 \\
\hline Sexo masculino & $30(45,5)$ & $9(90)$ & 1 \\
\hline \multicolumn{4}{|l|}{ Lugar } \\
\hline Pabellón general y sala de emergencias & $63(95,5)$ & $8(80)$ & 0,15 \\
\hline Unidad de Cuidados Intensivos & $18(27,3)$ & $10(100)$ & $0,015^{*}$ \\
\hline Infección adquirida en el hospital & $32(48,5)$ & $7(70)$ & 0,42 \\
\hline Criterio McCabe y Jackson & & & 0,22 \\
\hline No fatal & $38(57,6)$ & $3(30)$ & \\
\hline Rápidamente fatal y fatal como último caso & $27(40,9)$ & $8(80)$ & \\
\hline Tratamiento antimicrobiano adecuado & $65(98,5)$ & $2(20)$ & $0,014^{*}$ \\
\hline \multicolumn{4}{|l|}{ Enfermedades subyacentes } \\
\hline Hipertensión arterial & $10(15,2)$ & $2(20)$ & 0,19 \\
\hline Diabetes mellitus & $9(13,6)$ & $2(20)$ & 0,38 \\
\hline Enfermedad neurológica & $1(1,5)$ & $1(10)$ & 0,98 \\
\hline Cirrosis hepática & $2(3,0)$ & $2(20)$ & 0,8 \\
\hline Enfermedad biliar & $54(81,8)$ & $3(30)$ & 0,71 \\
\hline Trauma múltiple & 0 & $1(10)$ & 0,51 \\
\hline Cáncer con tumor sólido & $25(37,9)$ & $9(90)$ & 0,65 \\
\hline Neoplasia maligna hematológica & 0 & $1(10)$ & 0,59 \\
\hline \multicolumn{4}{|l|}{ Origen } \\
\hline Tracto biliar & $24(36,4)$ & $5(50)$ & 0,59 \\
\hline Catéter central & $3(4,5)$ & $4(40)$ & 0,11 \\
\hline Tracto urinario & $3(4,5)$ & $2(20)$ & 0,48 \\
\hline Tracto respiratorio & $2(3,0)$ & $1 \quad(10)$ & 0,12 \\
\hline Herida de piel o tejidos blandos & $2(3,0)$ & $1(10)$ & 0,33 \\
\hline Desconocido & $25(37,9)$ & $4(40)$ & 0,26 \\
\hline Microorganismo & & & 0,12 \\
\hline No-ERV & $50(75,8)$ & $8(80)$ & 0,69 \\
\hline ERV & $16(24,2)$ & $2(20)$ & 0,89 \\
\hline Bacteriemia polimicrobiana & $3(4,5)$ & $7 \quad(70)$ & $0,015^{*}$ \\
\hline
\end{tabular}

\begin{tabular}{|c|c|c|c|}
\hline \multirow[t]{2}{*}{ Variables testeadas $(n=76)$} & \multicolumn{2}{|c|}{$\begin{array}{c}\text { Modelo de regresión logística } \\
\text { multivariada }\end{array}$} & \multirow[t]{2}{*}{$\mathbf{p}$} \\
\hline & OR & $95 \% \mathrm{Cl}$ & \\
\hline Bacteriemia polimicrobiana & 1 & $0,83-4,3$ & 0,12 \\
\hline Tratamiento antimicrobiano adecuado & 0,33 & $0,14-0,79$ & 0,01 \\
\hline Ingreso a la Unidad de Cuidados Intensivos & 4,2 & $1,7-10$ & 0 \\
\hline Bacteriemia de origen no tracto biliar & 8,43 & $1,32-54,0$ & 0,002 \\
\hline
\end{tabular}

\section{Discusión}

Éste es el primer estudio que describe la incidencia de una ITS causada por E. faecium e identifica los factores de riesgo de mortalidad. Después de comparar nuestros resultados con otros estudios recientes de ITS por Enterococcus sp., pudimos apreciar que la proporción de ITS debidas a $E$. faecium fue similar a la suma total de episodios de ITS (6,8 vs 5,0-7,1\%). La proporción de una ITS debida a ERV, respecto de ITS causada por E. faecium, fue concordante con los resultados anteriores $(23,7 \%)$. De los 76 pacientes evaluados, sólo tres pacientes recibieron vancomicina parenteral antes de desarrollar una ITS por ERV, y ninguno recibió vancomicina por vía oral. 
Se han reportado las infecciones por ERV en animales utilizados como frente de alimentos como sucedió en la producción industrial de pollos. Como no ocurrió brote alguno, asumimos que no hubo una expansión clonal predominante en el año en que se desarrolló el estudio.

Entre nuestros pacientes, el rango de mortalidad a 30 días $(10 / 76 ; 13,2 \%)$ de una ITS por E. faecium fue más bajo de lo observado en estudios anteriores. Esta mortalidad más baja puede deberse a múltiples factores, incluyendo la población del paciente, variaciones en cuanto a puerta de entrada o condiciones subyacentes mayores y la ausencia de expansión de un clon letal. Se necesitan estudios futuros para dilucidar estos factores.

Una de las causas de ingreso más comunes en nuestros pacientes fue una enfermedad biliar $(38,2 \%)$, en la cual, con frecuencia, el drenaje adecuado alivió los síntomas clínicos. Una ITS por E. faecium con un origen no biliar fue un factor de riesgo para la mortalidad a 30 días; las cepas de E. faecium con un origen biliar fueron más frecuentemente susceptibles a vancomicina y fueron drenadas más fácilmente. Con todo lo expresado, nuestros resultados hacen hincapié a la necesidad de prestar una atención especial a los pacientes con una ITS por $E$. faecium con un origen no biliar, así como controlar la fuente de infección. Nuestros resultados concordaron con los principios internacionales del manejo de sepsis grave y de shock séptico.

El surgimiento de bacterias resistentes a antimicrobianos, incluyendo a ERV, es un complejo desafío para los médicos tratantes, quienes disponen de limitadas opciones terapéuticas. Los pacientes críticos ingresados a la UCI tienen un riesgo mayor de adquirir estas bacterias resistentes, lo que tendrá un impacto desfavorable en la mortalidad. El estudio de Jung mostró que la colonización de ERV está asociada al incremento de la mortalidad. Los resultados de nuestro estudio mostraron que la ITS presente al momento del ingreso de los pacientes a las UCI fue otro factor significativo de riesgo de mortalidad a 30 días. La estadía en la UCI (lo que puede reflejar la gravedad de la ITS) estuvo también fuertemente relacionada con las causas de muerte por ITS debida a E. faecium y ERV. Ciertamente, los pacientes críticos ingresados a la UCI están bajo un riesgo de mortalidad mayor y E. faecium tiene un rol importante como agente causal.

Dentro del rango de mortalidad a 30 días, un factor protector fue justamente una antibioterapia adecuada para la ITS por $E$. faecium $(\mathrm{OR}=0,33$; IC95\% = 0,14-0,79; $\mathrm{p}=0,013)$. En este respecto, nuestros resultados fueron similares a los de estudios anteriores. Cabe destacar que los resultados del presente estudio sugieren que un tratamiento antimicrobiano apropiado ayuda a bajar las probabilidades de mortalidad dentro del hospital. Los principios internacionales del manejo de sepsis grave y de shock séptico recomiendan la administración de antibioterapia de amplio espectro dentro de la primera hora del diagnóstico de shock séptico (1B) y de sepsis grave sin shock séptico (1C), siendo esto el objetivo del tratamiento. Por consiguiente, destacamos la necesidad de dar especial cuidado a los pacientes ingresados a la UCI con una ITS por E. faecium con origen no biliar, además de la necesidad de utilizar un tratamiento antimicrobiano adecuado con el propósito de sacar adelante a más pacientes con este tipo de infecciones.

El presente estudio tiene varias fortalezas. Lo más importante es que reunimos una serie completa de datos longitudinales, abarcando cuatro años. Tuvimos acceso a una excelente fuente de información para evaluar las características demográficas de las ITS causadas por E. faecium. Asimismo, estos hallazgos nos otorgan una información epidemiológica invaluable acerca de una ITS debida a ERV en Taiwán central.

Nuestro estudio, sin embargo, tiene varias limitaciones. Primeramente, debido a su naturaleza retrospectiva, la tipificación de las cepas aisladas no fue realizada por limitaciones de recursos técnicos; por lo tanto, la clonación y el genotipo de las cepas aisladas no pudieron ser evaluados. En segundo lugar, no se evaluó la susceptibilidad in vitro a daptomicina. Sader y cols., reportaron que todas las cepas aisladas de Enterococcus fueron susceptibles a daptomicina (CIM, $\leq 4 \mu \mathrm{g} / \mathrm{mL})$. En tercer lugar, evaluamos la utilidad de un tratamiento antimicrobiano utilizando los puntos de corte recomendados por NCCLS. El tratamiento para cepas de vancomicina con $\mathrm{CIM} \leq 32 \mu \mathrm{g} / \mathrm{mL}$ se consideró adecuado, ya existe, no obstante, información que sugiere que la resistencia puede ser inducible en algunas cepas. Por consiguiente, el tratamiento con vancomicina contra una infección debida a ERV-VanC, se ha considerado inadecuado. Más aún, no realizamos un análisis molecular por limitaciones en los recursos técnicos, asumimos que ocurrió un fenómeno similar en nuestra institución, como fuera comunicado con anterioridad.

Como conclusión, nuestros resultados sugieren que una ITS causada por E. faecium está comúnmente asociada con la enfermedad biliar, y a su vez, estar asociada con un riesgo de mortalidad menor. El rango de mortalidad debido a una ITS no biliar $(\mathrm{OR}=8,43$; IC95\% $=1,32$ $54,0, p=0,002)$ y el ingreso a la UCI $(\mathrm{OR}=4,2$; IC95\% $=1,7-10 ; p=0,002)$ fueron los únicos factores de riesgo de mortalidad significativos, dentro del rango de los 30 días. Un tratamiento antimicrobiano adecuado fue, en definitiva, un factor protector para la mortalidad a 30 días plazo $(\mathrm{OR}=0,33$; IC95\% $=0,14-0,79 ; \mathrm{p}=0,013)$. En consecuencia a todo lo mencionado en este estudio, destacamos la necesidad de prestar una especial atención a los pacientes ingresados a la UCI con ITS E. faecium de origen no biliar y la necesidad de utilizar un tratamiento 
antimicrobiano adecuado para ITS por E. faecium con el objetivo de recuperar a más pacientes con estas infecciones.

\section{Resumen}

Antecedentes: Las infecciones del torrente sanguíneo por Enterococcus faecium, particularmente aquellas causadas por enterococos resistentes a vancomicina (ERV), representan aún un desafío para los tratamientos. Este estudio está orientado a la evaluación de la mortalidad debido a la infección del torrente sanguíneo (ITS) por E. faecium y por enterococos resistentes a vancomicina (ERV) en Taiwán central. Materiales y Métodos: Analizamos de forma retrospectiva casos de ITS causadas por E. faecium genuinas en el Sistema del Hospital Changhua Christian, entre los días 1 de enero de 2010 y 31 de diciembre de 2013. Resultados: De los 76 casos analizados, 28 pacientes fueron ingresados a las Unidades de Cuidados
Intensivos (UCI) al comienzo de una ITS (36,8\%), 10 casos fueron asociados a bacteriemia polimicrobiana (13,2\%), y 29 casos tuvieron como puerta de entrada la vía biliar. En 18 casos se pudieron observar ERV (23,7\%). La mortalidad a 30 días fue de 13,1\% (10/76). El análisis multivariado mediante regresión logística mostró que la bacteriemia de origen no biliar $(\mathrm{OR}=8,43,95 \%$ intervalo de confianza $(95 \% \mathrm{CI})=1,32-54,00 ; \mathrm{p}=0,002)$, y el ingreso a la $\mathrm{UCI}(\mathrm{OR}=4,2 ; 95 \% \mathrm{CI}=1,7-10,0 ; \mathrm{p}=0,002)$, fueron factores de riesgo significativos para el rango de mortalidad de 30 días, así como un tratamiento de antimicrobiano apropiado constituye un factor protector en contra la mortalidad $(\mathrm{OR}=0,33 ; 95 \% \mathrm{CI}=0,14-0,79$; $\mathrm{p}=0,013)$. Conclusiones: Nuestros resultados destacan la necesidad de asistir a los pacientes ingresados a la UCI con ITS por E. faecium con origen no biliar. Hacemos énfasis a la aplicación de una antibioterapia adecuada para sacar adelante a un mayor número de pacientes con este tipo de infecciones.

\section{Referencias bibliográficas}

1.- Uttley A H, Collins C H, Naidoo J, George R C. Vancomycin-resistant enterococci. Lancet 1988; 103 (1): 57-8.

2.- LeClercq R, Perlot E, Duval J, Courvalin P. Plasmid-mediated resistance to vancomycin and teicoplanin in Enterococcus faecium. N Engl J Med 1988; 319 (3): 157-61.

3.- Ben R J, Lu J J, Young T G, Chi W M, Wang C C, Chu M L, et al. Clinical isolation of vancomycin-resistant Enterococcus faecalis in Taiwan. J Formos Med Assoc 1996; 95 (12): 946-9.

4.- National Nosocomial Infections Surveillance System. National nosocomial infections surveillance (NNIS) system report. data summary from January 1992 through June 2004, issued October 2004. Am J Infect Control 2004; 32 (8): 470-85.

5.- McDonald L C, Lauderdale T L, Shiau Y R, Chen P C, Lai J F, Wang H Y, et al. The status of antimicrobial resistance in Taiwan among grampositive pathogens: The Taiwan Surveillance of Antimicrobial Resistance (TSAR) programme, 2000. Int J Antimicrob Agents 2004; 23 (4): 362-70.

6.- Hsueh P R, Chen W H, Teng L J, Luh K T. Nosocomial infections due to methicillinresistant Staphylococcus aureus and vancomycin-resistant enterococci at a university hospital in Taiwan from 1991 to 2003 :

Resistance trends, antibiotic usage and in vitro activities of newer antimicrobial agents. Int $\mathbf{J}$ Antimicrob Agents 2005; 26 (1): 43-9.

7.- Conde-Estévez D, Grau S, Albanell J, Terradas R, Salvadó M, Knobel H. Clinical characteristics and outcomes of patients with vancomycin-susceptible Enterococcus faecalis and Enterococcus faecium bacteremia in cancer patients. Eur J Clin Microbiol Infect Dis 2011; 30(1):103-8. doi: 10.1007/s10096010-1029-5.

8.- Fortún J, Coque T M, Martín-Dávila P, Moreno L, Cantón R, Loza E, et al. Risk factors associated with ampicillinresistance in patients with bacteremia caused by Enterococcus faecium. J Antimicrob Chemother 2002; 50 (6): 1003-9.

9.- McBride S J, Upton A, Roberts S A. Characteristics and outcomes of patients with vancomycin-susceptible Enterococcus faecalis and Enterococcus faecium bacteraemia-a fiveyear retrospective review. Eur J Clin Microbiol Infect Dis 2010; 29 (1): 107-14. doi: 10.1007/ s10096-009-0830-5.

10.- Ghanem G, Hachem R, Jiang Y, Chemaly R F, Raad I. Outcomes for and risk factors associated with vancomycin-resistant Enterococcus faecalis and vancomycin-resistant Enterococcus faecium bacteremia in cancer patients. Infect Control Hosp Epidemiol 2007; 28 (9): 1054-9.

11.- Martínez-Odriozola P, Muñoz-Sánchez J, Gutiérrez-Macías A, Arriola-Martínez P, Montero-Aparicio E, Ezpeleta-Baquedano C, et al. Análisis de 182 episodios de bacteriemia por enterococo: Estudio de la epidemiología, microbiología y evolución clínica. Enf Infecc Microbiol Clin 2007; 25 (8): 503-7.

12.- Suppola J P, Kuikka A, Vaara M, Valtonen V V. Comparison of risk factors and outcome in patients with Enterococcus faecalis vs Enterococcus faecium bacteremia. Scand J
Infect Dis 1998; 30 (2): 153-7.

13.- Arias C A, Murray B E. The rise of the enterococcus: Beyond vancomycin resistance. Nat Rev Microbiol 2012; 10 (4): 266-78. doi: 10.1038/nrmicro2761.

14.- Treitman A N, Yarnold P R, Warren J, Noskin G A. Emerging incidence of Enterococcus faecium among hospital isolates (1993 to 2002). J Clin Microbiol 2005; 43 (1): 462-3.

15.- Chang C M, Wang L R, Lee H C, Lee N Y, Wu C J, Ko W C. Characterisation of vancomycin-resistant enterococci from hospitalised patients at a tertiary centre over a seven-year period. J Hosp Infect 2010; 74 (4): 377-84. doi: 10.1016/j.jhin.2009.10.025.

16.- Campanile F, Bartoloni A, Bartalesi F, Borbone S, Mangani V, Mantella A, et al. Molecular alterations of VanA element in vancomycin-resistant enterococci isolated during a survey of colonized patients in an Italian intensive care unit. Microbial Drug Resistance 2003; 9 (2): 191-9.

17.- Wang J, Chen Y C, Chang S C, Chen M L, Pan H J, Chang Y Y, et al. Control of vancomycin-resistant enterococci in a hospital: A five-year experience in a Taiwanese teaching hospital. J Hosp Infect 2004; 58 (2): 97-103.

18.- Tan C, Lai C C, Wang J Y, Lin S H, Liao C H, Huang Y T, et al. Bacteremia caused by nonfaecalis and non-faecium Enterococcus species at a medical center in Taiwan, 2000 to 2008. J Infect 2010; 61 (1): 34-43.

19.- Chang C, Wang L, Lee H, Lee N, Wu C, Ko W. Characterisation of vancomycin-resistant enterococci from hospitalised patients at a 
tertiary centre over a seven-year period. J Hosp Infect 2010; 74 (4): 377-84.

20.- Clinical and Laboratory Standards Institute. Performance standards for antimicrobial susceptibility testing. 16th Informational Supplement. CLSI document, M100-S16 CLSI, Wayne, PA (2006).

21.- Clinical and Laboratory Standards Institute. Methods for dilution antimicrobial susceptibility tests for bacteria that grow aerobically-Seventh ed: Approved standard M7-A7. 2007.

22.- McCabe W R, Jackson G. Gram-negative bacteremia. I. etiology and ecology. Arch Intern Med 1962; 110 (6): 847-55.

23.- Bone R C, Sibbald W J, Sprung C L. The ACCP-SCCM consensus conference on sepsis and organ failure. Chest 1992; 101 (6): 1484-3.

24.- Horan T C, Andrus M, Dudeck M A. CDC/NHSN surveillance definition of health care-associated infection and criteria for specific types of infections in the acute care setting. Am J Infect Control 2008; 36 (5): 309-32.

25.- Caballero-Granado F J, Becerril B, Cuberos L, Bernabeu M, Cisneros J M, Pachón J. Attributable mortality rate and duration of hospital stay associated with enterococcal bacteraemia. Clin Infect Dis 2001; 32 (4): 587-94.

26.- Peset V, Tallón P, Sola C, Sánchez E, Sarrión A, Pérez-Bellés C, et al. Epidemiological, microbiological, clinical, and prognostic factors of bacteremia caused by high-level vancomycin-resistant enterococcus species. Eur J Clin Microbiol Infect Dis 2000; 19 (10): 742-9.

27.- Clark N C, Teixeira L M, Facklam R R, Tenover F C. Detection and differentiation of vanC-1, van $\mathrm{C}-2$, and vanC-3 glycopeptide resistance genes in enterococci. J Clin Microbiol 1998; 36 (8): 2294-7.

28.- Zhao C, Sun H, Wang H, Liu Y, Hu B, Yu Y, et al. Antimicrobial resistance trends among 5608 clinical gram-positive isolates in China:
Results from the gram-positive cocci resistance surveillance program (2005-2010). Diagn Microbiol Infect Dis 2012; 73 (2): 174-81.

29.- Chang C M, Wang L R, Lee H C, Lee N Y, $\mathrm{Wu} \mathrm{C}$ J, Ko W C. Characterisation of vancomycin-resistant enterococci from hospitalised patients at a tertiary centre over a seven-year period. J Hosp Infect 2010; 74 (4): 377-84.

30.- Aslam M, Diarra M S, Checkley S, Bohaychuk V, Masson L. Characterization of antimicrobial resistance and virulence genes in Enterococcus spp. isolated from retail meats in Alberta, Canada. Int J Food Microbiol 2012; 156: 222-30.

31.- Xu J, Wang L, Wang K, Zhou Q. Eight-year surveillance of antimicrobial resistance among Enterococcus spp. isolated in the first bethune hospital. Physics Procedia 2012; 33: 1197-200.

32.- Nilsson O. Vancomycin resistant enterococci in farm animals-occurrence and importance. Infect Ecol Epidemiol 2012; 20: 3402.

33.- Shay D K, Maloney S A, Montecalvo M, Banerjee S, Wormser G P, Arduino M J, et al. Epidemiology and mortality risk of vancomycin-resistant enterococcal bloodstream infections. J Infect Dis 1995; 172: 993-1000.

34.- Suppli M, Aabenhus R, Harboe Z B, Andersen L P, Tvede M, Jensen J U. Mortality in enterococcal bloodstream infections increases with inappropriate antimicrobial therapy. Clin Microbiol Infect 2011; 17: 1078-83.

35.- Han S H, Chin B S, Lee H S, Jeong S J, Choi H K, Kim C O, et al. Vancomycin-resistant enterococci bacteremia: Risk factors for mortality and influence of antimicrobial therapy on clinical outcome. J Infect 2009; 58: 182-90.

36.- Dellinger R P, Levy M M, Rhodes A, Annane D, Gerlach H, Opal S M, et al. Surviving sepsis campaign: International guidelines for management of severe sepsis and septic shock, 2012. Intensive Care Med 2013; 39: $165-228$
37.- Cornejo-Juárez P, Vilar-Compte D, Pérez-Jiménez C, Namendys-Silva SA, Sandoval-Hernández S, Volkow-Fernández P. The impact of hospital-acquired infections with multidrug-resistant bacteria in an oncology intensive care unit. Int J Infect Dis 2015; 31 : 31-4.

38.- Jung E, Byun S, Lee H, Moon S Y, Lee H. Vancomycin-resistant enterococcus colonization in the intensive care unit: Clinical outcomes and attributable costs of hospitalization. Am J Infect Control 2014; 42: 1062-6.

39.- Cheah A L, Spelman T, Liew D, Peel T, Howden B P, Spelman D, et al. Enterococcal bacteraemia: Factors influencing mortality, length of stay and costs of hospitalization. Clin Microbiol Infect 2013; 19: e181-9.

40.- Sader H S, Flamm R K, Jones R N. Antimicrobial activity of daptomycin tested against gram-positive pathogens collected in Europe, Latin America, and selected countries in the Asia-Pacific region (2011). Diagn Microbiol Infect Dis 2013; 75: 417-22.

41.- National Committee for Clinical Laboratory Standards. Methods for dilution antimicrobial susceptibility tests for bacteria that grow aerobically. M7-A5 approved standard (5th ed.), NCCLS, Wayne, PA (2000).

42.- Billot-Klein D, Gutmann L, Sable S, Guittet E, van Heijenoort J. Modification of peptidoglycan precursors is a common feature of the low-level vancomycin-resistant VANB-type enterococcus D366 and of the naturally glycopeptide-resistant species Lactobacillus casei, Pediococcus pentosaceus, Leuconostoc mesenteroides and Enterococcus gallinarum. J Bacteriol 1994; 176: 2398-405.

43.- Lu C L, Chuang Y C, Chang H C, Chen Y C, Wang J T, Chang S C. Microbiological and clinical characteristics of vancomycin-resistant Enterococcus faecium bacteraemia in Taiwan: Implication of sequence type for prognosis. $\mathrm{J}$ Antimicrob Chemother 2012; 67: 2. 